\title{
Children's orientation judgments: Retinally or environmentally determined?
}

\author{
LILA GHENT BRAINE and CAROL KNOX \\ Barnard College, Columbia University, New York, New York 10027
}

\begin{abstract}
Children between the ages of 3 and 9 years judged the uprightness of pictures of realistic figures under three conditions: tilt of the visual surround, tilt of the subject, and tilt of both subject and surround in the same direction. The tilts used were 0,90 , and $180 \mathrm{deg}$. Judgments were not affected by tilt of the visual surround alone. With 90-deg head tilt, subjects of all ages made judgments in terms of gravitational cues. With 180-deg tilt, the youngest children made judgments based on retinal cues, whereas the older children used gravitational cues more often. The discussion made two main proposals: that uprightness judgments could be based on different cues under 90 - and 180-deg head tilts, and that the age differences were due to a change in the nature of the judgments being made rather than to a change in the framework used.
\end{abstract}

When one says that a shape is upright or that a line is vertical. these terms are not defined uniquely, but depend on the subject's trame of reference. If the heald is tilted, is the upright judged in terms of retinal cues or in terms of environmental cues? This question has been asked fiequently in traditional studies of adult perception, but only infrequently in developmental work or in studies of the physiological processes underlying perception. ${ }^{1}$ Rock and his colleagues (Rock, 1956; Rock \& Heimer, 1957; Rock \& Lcaman, 1963) have concluded that uprightness is determined by the subject's phenomenal frame of reference at a given moment, and that this frame of reference is determined ordinarily by environmental cues. This conclusion is supported, in general, by other work (Attneave \& Olson, 1967; Attneave \& Reid. 1968; Olson \&Attneave, 1970; also see reviews by Day \& Wade, 1969, and Howard \& Templeton, 1966), although it must be qualified in three ways.

First, environmental cues may be decisive for direct judgments of the uprightness of ordinary pictures, but retinal cues appear to be important for judgments that are inferred from the identification of ambiguous shapes. This distinction is particularly clear in Thouless (1947) work. More than $85 \%$ of his subjects, when standing with their heads between their legs, identified the shape (in an ambiguous figure) that was upright with respect to retinal cues, but only half the subjects used retinal cues in judging the orientation of the perceived tigure. Subsequent

This work was supported by Grant HD 05035 from the National Institutes of Health to the senior author. The authors would like to thank the students and teachers of St. Michael's Nursery School, the Oaks Nursery School, and the Isla Vista Elementary School, in Santa Barbara and Goleta, California. The results were reported at the meetings of the Eastern Psychological Association in Boston, 1972. Requests for reprints should be sent to L. G. Braine, Department of Psychology, Barnard College, Columbia University, New York, New York 10027. work has tended to assume that a judgment of the orientation of a shape is equivalent to the identitication of the shape (Gajzago \& Day, 1972; Rock, 1956; Rock \& Heimer, 1957), with confusing consequences. ${ }^{2}$

The second qualitication is that the amount of head tilt appears to influence the frame of reference. With tilts of 90 deg or less, which characterize a majority of the systematic studies, most responses are environmentally determined (Attneave \& Olson, 1967; Attneave \& Reid, 1968; Rock, 1956; Rock \& Heimer, 1957; Rock \& Leaman, 1963). With tilts of $180 \mathrm{deg}$, approximately half the subjects give responses in terms of retinal cues (Gajzago \& Day, 1972; Ghent, Bernstein, \& Goldweber, 1960; Kaniza \& Tampieri, 1968; Rock, 1956, Experiment VIII). The only direct comparison of the effects of 90 - and 180-deg tilts is in an experiment by Rock (1956), who found that the 90-deg tilt elicited significantly more environmentally determined responses than did the 180-deg tilt.

Finally, the age of the subject may be related to the use of different cues. Ghent et al. (1960) found that 9 of 10 preschool children judged the uprightness of pictures by using retinal cues, in contrast to the earlier findings of Rock and his colleagues with adult subjects. Tampieri (1968) claimed that preschool children used retinal cues in identifying an ambiguous figure more often than adults did, although the difference between the age groups was small. Recently, Gajzago and Day (1972) presented more compelling evidence of a developmental change: in response to a variety of different pictures, preschool children gave significantly more retinally deternined responses than adults did. Since all the developmental studies used 180-deg head tilt, the effects of age and of degree of tilt are confounded in the available literature.

The present study was concerned with two main 
Table 1

The Main Test Conditions

\begin{tabular}{cccc}
\hline \multirow{2}{*}{$\begin{array}{c}\text { Condi- } \\
\text { tion }\end{array}$} & $\begin{array}{c}\text { Age Groups } \\
\text { (Years) }\end{array}$ & \multicolumn{2}{c}{ Orientation of } \\
Head (Deg) & Room (Deg) \\
\hline A & $3-5$ & 0 & $0,90,180$ \\
B & $3,4,5,8-9$ & $0,90,180$ & 0 \\
C & $3,4,5,8-9$ & $0,90,180$ & $0,90,180$ \\
\hline
\end{tabular}

Vote-A subject was tested under Condition $A, B$, or $C$.

developmental questions. First. are orientation judgments the same for 90 - and 180-deg tilts. and doe's age interact with the degree of tilt? Second. does the environment affect orientation judgments by virtue of cues provided by the immediate visual surround. by gravitational cues, or by a combination of both? Does age interact with these cues? To separate these two kinds of cues. the stimuli were presented in a miniature room which could be tilted. This method was selected because it was assumed that a small room would enhance the salience of the visual surround cues by bringing them very close to the stimulus pictures. One difficulty in comparing the use of visual surround cues in different age groups is the possibility that younger children pay less attention to items in peripheral vision. and might be less likely to use environmental cues for this reason.

The experiment comprised three conditions (Table 1). For the subjects in Condition $\mathrm{A}$, the miniature room was tilted and the subject sat upright: for Condition $\mathrm{B}$. the miniature room remained upright and the subject was tilted: for Condition C. the miniature room and the subject were tilted the same way. The cues provided by the miniature room will be referred to as cues of the immediate visual surround. retinal cues will be those provided by the eve and retina, and gravitational cues will be the third set of cues assumed to be operating. The design of the experiment pits the cues against each other in various combinations: the immediate visual surround is. retinal and gravitational cues (Condition $\mathrm{A}$ ). retinal cues is. gravitational cues and the immediate visual surround (B). and gravitational cues is. retinal cues and the immediate visual surround $(C)$. Further. the role of these cues was evaluated for tilts of 90 and 180 deg.

\section{METHOD}

\footnotetext{
Apparatus

The miniature room was a cube. $53.3 \mathrm{~cm}$ on a side, furnished to provide many cues to the upright. A sofa and coffee table were centered against the back wall. To the viewer's left were an armchair. with a doll sitting in it. and a set of shelves, with a telephone and a vase of flowers on the top shelf. To the right were a small table and two chairs. A rug was on the floor, and a light fixture in the ceiling illuminated the room. Everything in the room was fastened into place so that nothing would move when the room was tilted.

In the center of the wall facing the subject was a $10.2-\mathrm{cm}$-square aperture through which the subject could view the interior of the
}

room and the stimuli to be judged. Pairs of stimuli were presented in two $7.6-\mathrm{cm}$-square frames. separated by $3.8 \mathrm{~cm}$; these frames were located on the back wall and were centered with respect to the horizontal and vertical dimensions of the wall. The back wall was removable. and could be placed so that the picture frames were side by side or one above the other. i.e.. horizontal or vertical with respect to gravity. These arrangements of frames were compared to see whether any of the effects of the 90 -deg tilt could be attributed to a change in the apparent placement of the two stimuli, from a horizontal arrangement to a vertical arrangement, or vice versa.

\section{Tult Conditions}

Miniature room tilt only (Condition $A$ ). The room rested on a small table, and in front of the viewing wall was a headrest. The subject sat on a chair. and placed his chin on the headrest in order to look through the viewing aperture. The room was presented at 0 -deg tilt first to all subjects, and then at $90-\mathrm{deg}$ tilt (left and right) and 180 -deg tilt, in a counterbalanced order. The tilting of the room was done in full view of the subject.

Subject tilt only (Condition B). In general, the miniature room rested on the floor throughout testing. For the 0 -deg subject tilt, the subjects lay prone on the floor. and placed chin and hands on the headrest in order to look through the viewing aperture. For the 90 -deg tilt. the subjects lay prone on a platform. and turned the head to look in the viewing aperture. thus achieving a full 90.deg tilt. (In the early part of the testing, some subjects sat up and tilted their heads $90 \mathrm{deg}$; the data for the sitting and lying conditions were combined since the responses were the same.) Half the subjects were tilted $90 \mathrm{deg}$ to the left, and the other half, $90 \mathrm{deg}$ to the right. For the 180 -deg tilt, the subjects lay prone on the roof of the room, and lowered their heads to peek in through the viewing aperture, with head and hands on the headrest.

Combined subject and room tilt (Condition $\mathrm{C}$ ). The subjects were tilted in the manner described in the preceding section. and the miniature room was tilted in the same direction, and to the same degree, as the subject.

\section{Stimuli}

The stimuli consisted of pairs of pictures of different, mono-oriented objects. Thirty different pairs were constructed from 18 items: man, flowers, face, boat, clown, car, cat, cow, table, house, boy, tricycle. telephone. truck. girl, horse, chair. shoe. For the 0 -deg tilt of the subject and the room. one member of the pair was upright and one was inverted. For the various conditions of tilt. one picture was upright with respect to gravity, and the other was upright with respect to the object being tilted (person or miniature room). Under all conditions, the subject's task was to name the member of the pair that was turned the wrong way, was "upside-down."

\section{Procedure}

One experimenter sat behind the apparatus and inserted the stimuli for each trial. while another experimenter sat with the subject to record responses and to instruct the subject. The subject was invited to look through the aperture into the miniature room. and to name or describe some of the things in it. The tirst pair of stimuli was already in place. appearing as pictures on the back wall of the room, and the subject was told that the task was to name the picture that was turned the wrong way. that was "upside-down."

For the first six trials for all subjects, the subject and the room were both upright. Lnder this condition only, errors were corrected. and additional trials were given until the subject made six consecutive correct responses. (Only a few of the youngest subjects required additional trials). Twelve trials were presented under each of the two tilt conditions ( 90 and $180 \mathrm{deg}$ ). making a total of 30 trials for each subject. Between each trial. the subjects closed their eves. and were told to open them when the next pair of stimuli was in place.

Within an age-sex category for each of the main conditions (A. B, C). the subjects were assigned randomly to balance the following variables: the arrangement of the stimulus frames (vertical or 
horizontal), the order of tilts ( 180 or 90 deg tirst), the direction of tilt for the 90-deg condition (left or right). and the order of trials (1-12 or $12-1)$. An equal number of boys and girls was tested at each age under each condition, except for the group in the room-tilt condition, for reasons to be described in the results section.

\section{Identification of Expression}

For the third-grade subjects only, two additional trials were run under conditions of 180 -deg head tilt to assess the effect of head tilt on identitication of expression. The subjects were shown an ambiguous face (Kanizsa \& Tampieri, 1968), which had an angry expression in one orientation and a happy expression when rotated $180 \mathrm{deg}$. Each subject saw each orientation of the face (the order being balanced across subjects) and was required to describe the facial expression.

\section{Subjects}

There were 35 children of 3 years, 42 of 4 years, 51 of 5 years, 48 of 8-9 years of age, with a nearly equal number of boys and girls in each age group. The preschool children came from two schools serving predominantly upper middle-class parents, and the older children were drawn from a public school serving families from low to upper middle class.

\section{RESULTS}

The judgments made by the groups from 3 to 5 years will be described in turn for miniature room tilt. subject tilt. and combined room and subject tilt. The results for the subjects of $8-9$ years will be reportec separately, for reasons to be described later. After the main effects due to tilt condition and age have been presented. the effects of other variables will be considered briefly.

\section{Miniature Room Tilt}

Testing under this condition was terminated after the tirst 16 subjects ( 3 subjects of 3 years, 10 of 4 years. and 3 of 5 years). since the room tilt alone did not affect judgments. For both the 90-deg tilt and the 180-deg tilt. all but one of the 16 subjects on every trial selected as $w$ rong the figure that was disoriented with respect to retinal and gravitational cues. The exception was a subject of 4 years, who, on one trial under $180-\mathrm{deg}$ tilt, first reported that both pictures were upright. but when pressed to select one that was a little bit wrong, she disregarded the cues from the visual surround.

\section{Subject Tilt}

A subject was categorized as making either retinal or gravitational judgments if the responses on 9 of the

Table 2

Percentage of Subjects Making Gravitational (G), Retinal (R), or Inconsistent (I) Judgments Under Conditions of Head Tilt

\begin{tabular}{|c|c|c|c|c|c|c|c|}
\hline \multirow{2}{*}{$\begin{array}{c}\text { Age } \\
\text { (Years) }\end{array}$} & \multirow[b]{2}{*}{$\mathrm{N}$} & \multicolumn{3}{|c|}{ 90-Deg Tilt } & \multicolumn{3}{|c|}{ 180-Deg Tilt } \\
\hline & & G & $\mathbf{R}$ & I & G & $\mathrm{R}$ & I \\
\hline 3 & 16 & 94 & 0 & 6 & 6 & 50 & 44 \\
\hline 4 & 16 & 100 & 0 & 0 & 37 & 44 & 19 \\
\hline 5 & 32 & 94 & 3 & 3 & 53 & 34 & 13 \\
\hline
\end{tabular}

Table 3

Percentage of Subjects Making Gravitational (G), Retinal (R), or Inconsistent (I) Judgments Under Conditions of Combined Head and Room Tilt

\begin{tabular}{|c|c|c|c|c|c|c|c|}
\hline \multirow{2}{*}{$\begin{array}{c}\text { Age } \\
\text { (Years) }\end{array}$} & \multirow[b]{2}{*}{$\mathrm{N}$} & \multicolumn{3}{|c|}{ 90-Deg Tilt } & \multicolumn{3}{|c|}{ 180-Deg Tilt } \\
\hline & & $G$ & $\mathrm{R}$ & I & $\mathrm{G}$ & $\mathrm{R}$ & I \\
\hline 3 & 16 & 88 & 0 & 12 & 31 & 50 & 19 \\
\hline 4 & 16 & 88 & 0 & 12 & 19 & 62 & 19 \\
\hline 5 & 16 & 94 & 6 & 0 & 25 & 62 & 13 \\
\hline
\end{tabular}

12 trials were based on the same cues; the subject was categorized as inconsistent if the responses were distributed less systematically.

With 90-deg tilt, 61 of the 64 subjects between 3 and 5 years judged the upright in terms of gravitational cues, 1 subject used retinal cues, and 2 subjects were inconsistent (Table 2). Of the 61 subjects making gravitational judgments, 56 subjects made this judgment on all 12 trials.

With 180-deg tilt, judgments showed considerable variability within and across age groups (Table 2). Judgment type changed with age (chi square = 12.442, $\mathrm{df}=4 . \mathrm{p}<.02$ ), with the subjects of 3 years using retinal cues and the older subjects shifting towards the use of gravitational cues. The inconsistent responses shown by many of the young children reflected a specific difficulty with the vertical arrangement of the stimuli (as will be discussed shortly), and not a general difficulty in making an orientation judgment.

\section{Combined Room and Subject Tilt}

Again, subjects were categorized as making gravitational or retinal judgments, or as being inconsistent, using the same criteria as for the subject tilt alone. With 90-deg tilt, gravitational judgments were made $t y$ a high proportion of the subjects in each age group (iable 3). In analyzing the consistency of response over the 12 trials, there was lesser consistency than that shown by the subjects tested with 90-deg tilt of the subjects only (chi square $=$ $8.55 . \mathrm{df}=1, \mathrm{p}<.01$ ), suggesting that the tilt of the visual surround contributed some uncertainty, but did not affect the basic judgments. With 180-deg tilt, a majority of the subjects in each age group made retinal judgments, and to approximately the same degree at each age. Again, the deviant performance in the 3-year-old group was contributed by those subjects presented with the vertical arrangement of the stimuli; when only the horizontal presentation is considered. 7 of the 8 subjects made retinal judgments and 1 was inconsistent.

\section{Children of 8.9 Years}

These results are presented separately because we had the impression. from the subjects' spontaneous verbalizations. long hesitations. and the judgments 
Table 4

Percentage of Third-Grade Subjects Making Gravitational (G), Retinal (R), or Inconsistent (I) Judgments

\begin{tabular}{lccccccccc}
\hline & & \multicolumn{3}{c}{$90-$ Deg Tilt } & & \multicolumn{3}{c}{ 180-Deg Tilt } \\
\cline { 3 - 5 } \cline { 7 - 9 } Tilt & N & G & R & I & & G & R & I \\
\hline Head Only & 32 & 81 & 16 & 3 & & 41 & 53 & 6 \\
Head and Room & 16 & 75 & 25 & 0 & 31 & 50 & 19 \\
\hline
\end{tabular}

themselves, that the older subjects were aware of the possibility of making a judgment on more than one basis and seemed in contlict as to how to do the task. One subject said, "It's hard to decide which way to do it," and another said, "It looks upside-down, but it is really rightside-up." Not one of the 112 children between 3 and 5 years made remarks of this sort.

Like the younger children, the subjects of 8-9 years made predominantly gravitational judgments under 90-deg head tilt; nevertheless, 9 of the 48 subjects in this group made retinal judgments. By contrast, only 2 of the 112 subjects between 3 and 5 years of age made retinal judgments (comparing the younger and older subjects, chi square $=12.57, \mathrm{p}<.01$ for $1 \mathrm{df}$ ). Under conditions of $180-\mathrm{deg}$ tilt, about half the subjects of 8-9 years made head judgments, and the incidence of this judgment was not influenced by the orientation of the visual surround.

\section{Stimulus Arrangement}

The judgments elicited under 90-deg tilt were impervious to any effect of stimulus arrangement. Under 180-deg tilt, the vertical and horizontal presentations of stimulus pairs yielded different distributions of responses in the group of 3 years (chi square $=13.85, \mathrm{p}<.01$ for $2 \mathrm{df}$ ), and nonsigniticant chi squares in the older groups. In general, the vertical presentation appeared to confuse the young children, which is consistent with evidence showing that a vertical arrangement of stimuli makes the discrimination of upright from inverted difficult for children of this age (e.g., Barroso \& Braine, 1974).

\section{Sex Differences}

With 180-deg head tilt, girls tended to make more retinal judgments than boys (except in the 3-year-old group, where most subjects gave retinal judgments). Table 5 shows the distribution of judgments for boys and girls. combining the data across all age groups and all conditions involving $180-\mathrm{deg}$ subject tilt (chi square $=9.71 . \mathrm{p}<.01$ for $2 \mathrm{df}$ ). The girls' tendency to make head judgments is not a general tendency to use retinal or "egocentric" cues, since most of the girls gave gravitational judgments under conditions of $90-\mathrm{deg}$ tilt. Of the subjects giving retinal judgments with 90 -deg head tilt ( 2 subjects of 5 years and 9 of $8-9$ years), 5 were girls and 6 were boys.

\section{Ambiguous Figure}

As described in the procedure, the subjects of 8-9 years were asked to identify, while tilted $180 \mathrm{deg}$, the expression of an ambiguous figure. Eight-four percent of the subjects used retinal cues in identifying the expression, similar to earlier findings with this figure (Kanizsa \& Tampieri, 1968; Tampieri, 1968). Of main interest, however, are the 13 subjects who gave judgments of orientation (during the main task) that were based on the gravitational framework: 12 of these subjects gave identifications of expression based on retinal cues. As suggested in the introduction, identification of an ambiguous figure may involve the use of different cues than a judgment of orientation.

\section{DISCUSSION}

\section{Visual Surround}

Tilt of the visual surround alone was without discernible effect in the age groups tested (3-5 years), and tilt of the surround in conjunction with body tilt added only slightly to the effect of body tilt. Although earlier work (e.g., Asch \& Witkin, 1948) found that the visual surround influenced orientation judgments, procedural differences between this and previous studies could account for the different results. First. the subjects in this study gave judgments of uprightness rather than judgments of verticality. Second, the earlier procedures usually provided an adaptation period of several minutes, whereas the present study had no adaptation period and only 12 test trials (for each tilt). Finally, it may have been easier to disregard the visual surround in the present experiment because of the unnaturally small size of the room. Despite these qualifications, it seems reasonable to conclude that cues from the visual surround play a minimal role in judgments of uprightness.

\section{Comparison of 90- and 180-deg Tilts}

The most striking result of the present study was the contrast between the effects of the two head tilts. With 90-deg tilt, most subjects used gravitational cues, whereas with $180-\mathrm{deg}$ tilt, either retinal or

Table 5

Number of Females and Males Making Gravitational (G), Retinal (R), or Inconsistent (I) Judgments for All Age Groups Combined

\begin{tabular}{lrrrrrrr}
\hline & \multicolumn{3}{c}{ Female } & & \multicolumn{3}{c}{ Male } \\
\cline { 2 - 6 } \cline { 5 - 8 } Tilt (180 deg) & G & R & I & & G & R & I \\
\hline Head Only & 12 & 25 & 11 & & 25 & 19 & 4 \\
Head and Room & 6 & 20 & 6 & 11 & 16 & 5 \\
Total & 18 & 45 & 17 & 36 & 35 & 9 \\
\hline
\end{tabular}


gravitational cues were used. A difference between 90 and 180-deg tilts might have been anticipated for two reasons.

First, the structure and function of the human body predispose us towards 90 -deg tilts. The head is so connected to the body that the head can tilt $90 \mathrm{deg}$ without changing body position; further, we assume daily a position with the head and body at a $90-\mathrm{deg}$ angle to the gravitational vertical. By contrast, the head cannot swivel $180 \mathrm{deg}$ on the neck, nor are we likely to assume a position which inverts head and body. Both structurally and experientially, the human organism develops so that the perception of visual space does not change every time we tilt our heads. In fact, there is evidence for tilt compensation that is mediated directly (in the cat) by some of the orientationally selective cells of the visual cortex (Horn \& Hill, 1969) and of the superior colliculus (Bisti, Maffei, \& Piccolino, 1972). Despite the plausibility of an organismic explanation for the difference between the effects of 90- and 180-deg tilts, a second factor must be considered.

The task requirements are different for the two degrees of tilt. In the 90-deg task, the subject must choose between figures at right angles to each other, one aligned with the gravitational vertical, the other with the retinal vertical. The evidence from our daily experience, as well as from the laboratory (Attneave \& Olson, 1967; Gibson, 1952; Howard \& Templeton, $1966)$, indicates that the phenomenal vertical is determined by gravitational rather than retinal cues. For young children, the phenomenal vertical is a salient cue to the upright (Ghent, 1961). Thus some subjects may judge uprightness simply by judging whether the axis connecting the top and bottom features of the figure is parallel to the phenomenal vertical, and this judgment is based on gravitational cues. With 180-deg tilt, however, the phenomenal vertical cannot serve as a cue since both figures appear vertical. To choose one of the figures as upright. the subject must judge whether the feature detining the top of the figure (e.g., the head of the clown) is appropriately located in space. This analysis suggests that different cues are used for different tilts: alignment with the phenomenal vertical for the $90-\mathrm{deg}$ tilt, and the location of the feature defining the top of the figure, for the 180-deg tilt.

\section{Change with Age}

With 180-deg head tilt, the consistent responses of the 3-year-old subjects were based on retinal cues in almost all cases, whereas about half the subjects of 5 years and of 8-9 years based their responses on gravitational cues. This developmental shift agrees with previous data for 180 -deg tilt in children (Gajzago \& Day, 1972; Ghent et al., 1960; Tampieri, 1968). In adult groups tested with 180-deg tilt, approximately half the subjects also made orientation judgments based on gravitational cues (Rock, 1956; Thouless, 1947).

The age change observed during the preschool period might be interpreted as a shift in the framework used for defining space. That is, older children and adults use objective space, or the gravitational frame of reference, whereas young children use an egocentric frame of reference, the head and retina. There are at least two difficulties with this interpretation. First, under 90 -deg tilt, the young children did not use the retinal framework, and increasing age was associated with the increasing use of retinal cues in this condition. Second, the age change (with 180-deg tilt) is peculiarly incomplete, since about half the older subjects continue to make retinal judgments. It is implausible to claim that these subjects do not know where the top of the real world is located when their heads are tilted $180 \mathrm{deg}$, i.e., that their phenomenal visual space is defined by retinal cues.

An alternative interpretation is that it is the orientation judgment that changes with age, rather than the framework. Ordinarily, uprightness is understood to refer to the relation between a figure and a framework: the feature defining the top of the figure (e.g., the head of a clown) appears at the top of the phenomenal spatial framework. Another possibility is that uprightness is based on whether the figure "looks familiar" or "looks right" in contrast to "looking strange." Kohler (1940) implied such a distinction between a framework judgment and a "looking right" judgment when he claimed (while standing with his head between his legs) that a face "looked strange" when upside-down with respect to his head, although he judged the face to be upright in space (p. 32-33). (Conversely, the figure judged to be upside-down in the real world was perceived by Kohler as having its usual appearance when upright with respect to the head.) Thouless (1947) also distinguished between an optimal perceptual organization for an ambiguous figure (based on retinal cues for 180 -deg tilt) and a judgment of the orientation of the perceived figure.

Assume that the preschool child judges uprightness almost exclusively on the basis of whether or not the tigure "looks familiar or right" and that this judgment is based on retinal cues (for 180-deg tilt). Around 5 years of age, the child becomes able to make the orientation judgment relating the figure to the spatial framework. From this age upwards, some people judge uprightness in terms of the "familiar" or "right" appearance of the figure, whereas other people make the framework judgment; the incidence of each judgment in older subjects would depend on a variety of factors (e.g., cues from the visual surround). For 90 -deg tilts, the young child also makes a "looks right" judgment which, in this case, is 
based on the cue of the apparent vertical. In the older child, the use of the apparent vertical cue declines. and the "looks right" judgment is based on retinal cues. For both tilts, then, the young child judges uprightness in terms of the "looks familiar or right" judgment. whereas the older subject uses either a framework judgment or a "looks" judgment ${ }^{3}$; the framework judgment is based on gravitational cues at all ages, whereas the cues for the "looks" judgment change with age.

A basis for the judgment that a figure "looks right" in one orientation rather than another has already been proposed for geometric shapes (Braine, 1972, 1973: Ghent. 1961): the analogous judgment for realistic figures can be accounted for by a variation of the earlier proposal. Assume that the serial processing of the features (Hebb. 1949; Neisser, 1967) of a two-dimensional shape is not random, but starts at the top (with respect to retinal cues) and continues in a dow nwards direction (Braine. 1972). For realistic figures that have a usual orientation in space, the parts of the figure would tend to be processed in a particular order. and in the same order on different occasions. A picture could be judged as looking "right" or "familiar" when the parts of the figure are processed in a familiar order. An alternative way of looking at the link between recognition processes and the "familiarity" judgment is to focus directly on constructs in menory. Earlier work suggested that representations of mono-oriented figures are stored in long-term memory in the upright orientation (Braine, 1973). A mismatch between the input and the representation would provide the basis for perceiving the input as wrong or unfamiliar; comparison between the input and the representation would involve a sampling of parts tagged with the same spatial location. ${ }^{4}$ These two bases could be seen as two parts of one process if the serial processing of features were assumed to provide the orientation information required for the representation.

\section{Recapitulation}

The position developed here is that an uprightness judgment can be made on two bases: a perception that a tigure "looks right or familiar" or an evaluation that a figure is oriented appropriately with respect to a framework. In general, the literature is compatible with such a view. First, the analysis of the cues underlying the different judgments provides an explanation for the different results obtained with 90 and 180-deg tilts. Second, the behavior of the subjects of different ages can be accounted for by assuming that young children, unlike older subjects, are limited to the "looks right" judgment. Third, when ambiguous figures are used as stimuli, the figure perceived is based on the "looks right" judgment, whereas the orientation of the perceived figure may be judged either on the basis of appearance or by relating the figure to the framework. Finally, the shifts observed between retinal and environmental frameworks, often without apparent cause (Attneave \&Reid, 1968; Olson \& Attneave, 1970), could be plausibly interpreted as shifts between the two types of judgment, and such shifts would be related to the nature of the stimuli and to voluntary control exerted by the subject. The framework judgment need not be limited to the use of the gravitational frame of reference. as the work of Rock and Leaman (1963) has shown.

\section{REFERENCES}

Asch, S. E., \& Witkin, H. A. Studies in space orientation. II Perception of the upright with displaced visual fields and with body tilted. Journal of Experimental Psychology, $1948,38,455-477$.

Attneave, F., \& Olson, R. K. Discriminability of stimuli varying in physical and retinal orientation. Journal of Experimental Psychology, 1967, 74, 149-157.

Attheave, F., \& Reid, K. W. Voluntary control of frame of reference and slope equivalence under head rotation. Journal of Experimental Psychology, 1968, 78, 153-159.

Barroso, F., \& Braine, L. Ghent. "Mirror-image" errors without mirror-image stimuli. Journal of Experimental Child Psychology, 1974, 18, 213-225.

Bisti, S., Maffei, L., \& Piccolino, M. Variations of the visual responses of the superior colliculus in relation to body roll. Science, 1972, 175, 456-457.

Braine, L. Ghent. A developmental analysis of the effect of stimulus orientation on recognition. American Journal of Psychology, 1972, 85, 157-187.

Braine, L. Ghent. Perceiving and copying the orientation of geometric shapes. Journal of Research and Development in Education, 1973, 6, 44-55.

DAY, R. H., \& WADE, N. J. Mechanisms involved in visual orientation constancy. Psychological Bulletin, 1969, 71, 33-42.

GajzaGo, C., \& DAY, R. H. Uprightness constancy with head inversion in young children and adults. Journal of Experimental Child Psychology, 1972, 14, 43-52.

Ghent, L. Form and its orientation: A child's-eye view. American Journal of Psychology, 1961, 74, 177-190.

Ghent, L., Bernstein, L., \& Goldweber, A. M. Preferences for orientation of form under varying conditions. Perceptual and Motor Skills, 1960, 11, 46.

Gibson, J. J. The relation between visual and postural determinants of the phenomenal vertical. Psychological Review, 1952, 59, 370-375.

HeBs, D. O. The organization of behavior. New York: Wiley, 1949.

Horn, G., \& HiLl, R. M. Modifications of receptive fields of cells in the visual cortex occurring spontaneously and associated with bodily tilt. Nature, 1969, 221, 186-188.

Howard, I. P., \& Templeton, W. B. Human spatial orientation. New York: Wiley, 1966.

Kanizsa, G., \& Tampieri, G. Nuove osservazioni sull'orientamento retinico ed ambientale. In Ricerche sperimentali sulla percezione, publicato a cura dell'Ist. di Psicologia dell'Università di Trieste, 1968.

Koнler, W. Dynamics in psycho'ogs. New York: Liveright, 1940.

NeIsser, U. Cognitive psychology. New York: Appleton-CenturyCrofts, 1967.

Olson, R. K.. \& Attneave, F. What variables produce similarity grouping? American Journal of Psychology, 1970 , 83. 1.21. 
Rock, I. The orientation of forms on the retina and in the environment. American Journal of Psychology, 1956, 69, 513-528.

Rock, I. The nature of perceptual adaptation. New York: Basic Books, 1966

Rock, I., \& HeImer, W. The effect of retinal and phenomenal orientation on the perception of form. American Journal of Psychology, 1957, 70, 493-511.

Rock, I., \& LeAman, R. An experimental analysis of visual symmetry. Acta Psychologica, 1963, 21, 171-183.

TAMPIE RI. G. L'influenza dei sistemi di riferimento ambientale e retinico sull'organizzazione percettiva nei bambini. Rivista di Psicologia, 1968, 62. (No. 2), 1-8.

Thouless, R. H. The experience of "upright" and "upsidedown" in looking at pictures. In Miscellanea Psychologica a Albert Michotte. Louvain: University of Louvain, 1947.

\section{NOTES}

1. In work on orientation-specific cells in the visual system, the assumption appears to be made that the orientation to which the cell is responsive is defined with respect to retinal position, although two recent studies (Bisti, Maffei, \& Piccolino, 1972; Horn \& Hill, $1969)$ have reported that some cells do not fit this assumption.
2. For example, Gajzago and Day (1972) investigated "uprightness constancy" (stability of orientation judgments with the head tilted), using pictures of both ordinary objects and ambiguous figures. They concluded that uprightness constancy was better for ordinary objects than for ambiguous figures, since ordinary objects were judged with respect to gravity more often than ambiguous figures were so judged. However, an alternate interpretation is that the subjects made different responses to the two types of figures: an orientation judgment for the ordinary figures and an identification response for the ambiguous figures.

3. The subjects of 8-9 years indicated that the task instructions were ambiguous, and the present analysis suggests that the ambiguity would be clarified by asked either for judgments of uprightness in the real world or for judgments of whether the figure "looks right." These instructions should elicit the same responses in children between 3 and 4 years of age, but different responses in older children.

4. The implicit assumption here is that "top" for the internal representation is defined retinally; a somewhat similar assumption was made by Rock (1966) in accounting for the observation that the world looked upside-down when the retinal image was rotated $180 \mathrm{deg}$ from the usual position.

(Received for publication July 12, 1974; revision received February 3,1975 .) 